DGfK-Nachrichten

\section{DGfK-Präsident: \\ Aus der Arbeit des Vorstandes}

Liebe DGfK-Mitglieder,

liebe Leserinnen und Leser, in der Deutschen Gesellschaft für Kartographie hat sich seit unserem Jubiläums-DKT zum 60. Geburtstag der DGfK in Nürnberg im Jahr 2011 eine sehr offene und ebenso konstruktive Diskussion über die Zukunft der deutschen Kartographie und der DGfK entwickelt. Die DGfK neu auszurichten, die aktuelle Bedeutung der Kartographie in unsere Fachgesellschaft, in Nachbarverbände und in die Öffentlichkeit zu kommunizieren und als Folge der Neuorientierung unseren Vereinsnamen zu erweitern dies alles wird in den Sektionen und Gremien der DGfK intensiv diskutiert und voran getrieben.

Überlegungen, die Kommunikation raum-zeitlicher Informationen, also die Geokommunikation, im weiteren Sinne stärker in die Definition der Kartographie aufzunehmen, um so neben der Visualisierung auch akustische und haptische Präsentationsformen zu berücksichtigen, stehen dabei im Einklang mit dem Anliegen, die seit jeher hohen Ansprüche der Kartographie an Gestaltung und Darstellung von Geodaten zu gewährleisten. Darstellung und Kommunikation raum-zeitlicher Informationen

- damit lässt sich sehr treffend und zeitgemäß die Disziplin Kartographie definieren. Und damit ist auch das weite Arbeitsgebiet derjenigen beschrieben, die kartographisch tätig sind, ob sie nun eine Ausbildung oder ein Studium der Kartographie absolviert oder die Kartographie auf anderem Wege erlernt haben.

Kartographie als Alleinstellungsmerkmal, als zentraler und Identität stiftender Begriff unserer Fachgesellschaft und als eingeführte Marke in Fachkreisen und in der Öffentlichkeit

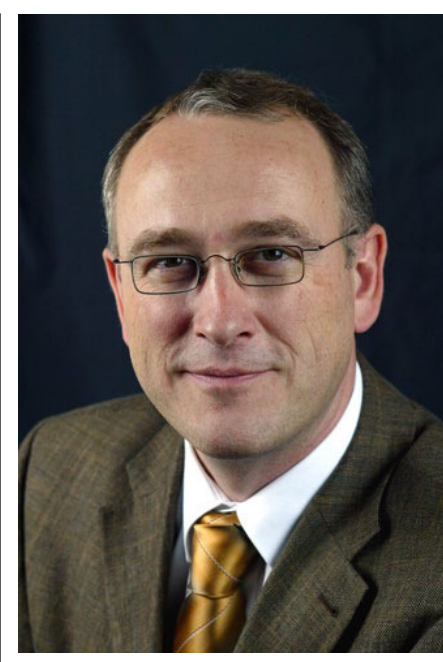

Prof. Dr. Manfred Weisensee

wurde in großer Übereinstimmung im Rahmen des 60. DKT in Hannover durch die Gremien der DGfK bestätigt. Gleichermaßen bestätigt wurde aber auch die Offenheit der DGfK für alle in der Kartographie tätigen Berufe. Der Vorstand wurde beauftragt, die strategische Weiterentwicklung der DGfK voranzutreiben. In einer gemeinsamen Sitzung von Vorstand und Sektionsvorständen in Berlin wurde im Januar dieser Auftrag der Mitgliederversammlung zur Vorbereitung von Beschlüssen der Gremien umgesetzt. Ergebnis der Strategiesitzung ist der in der Einladung zur Mitgliederversammlung, siehe Seite $X$, enthaltene Beschlussantrag des Vorstandes, den Namen unserer Fachgesellschaft zu ändern in Deutsche Gesellschaft für Kartographie e.V. (DGfK) - Gesellschaft für Kartographie und Geomatik. Dass damit die Diskussion nicht abgeschlossen ist, zeigen uns zahlreiche Beiträge aus den Sektionen. Und wir freuen uns darauf, die Diskussion bis zur Mitgliederversammlung im Rahmen des 61. DKT in Dresden fortzuführen.

Ein wichtiger Tagesordnungspunkt der diesjährigen Mitgliederversammlung ist die Wah/ des Vorstands der DGfK; den Wahlaufruf des Wahlleiters Fritz Meier finden Sie auf Seite X. Leider wird sich unser Vizepräsident national Thomas Harder aus gesundheitlichen Gründen nicht wieder zur Wahl stellen. Auch an dieser Stelle sei inm für seine Bereitschaft und sein Engagement für die DGfK in der Sektion und im Vorstand herzlich gedankt. Vor diesem Hintergrund bitte ich Interessentinnen und Interessenten an einer Mitarbeit im Vorstand ganz besonders, sich bei unserem Wahlleiter, den Sektionsvorständen oder einem Vorstandsmitglied zu melden.

Ich möchte es auch nicht versäumen, Sie noch einmal zu unserem ganz besonderen diesjährigen Deutschen Kartographentag (vom 28.-30. August) einzuladen, der in enger Verbindung mit der 26. Konferenz der Internationalen Kartographischen Vereinigung (vom 25.-30. August) in der Kongressstadt Dresden stattfinden wird. Die Anmeldung zu beiden Veranstaltungen ist über die Internet-Präsenz der DGfK und unter www.kartographentag.net erreichbar und der Frühbuchertarif wurde bis zum 15. April verlängert. Weiterhin wurde vereinbart, dass über die Sektionsleitungen besondere Gruppenfahrten zu DKT und ICC organisiert werden. Zur Intensivierung der Zusammenarbeit mit den befreundeten Geofachverbänden können auch die Mitglieder von DGPF, DVW und VDV zu besonderen Konditionen am diesjährigen DKT teilnehmen.

Manfred Weisensee, Hude

Präsident der DGfK

\section{Aufruf zur}

\section{DGfK-Vorstandswahl 2013}

Der Wahlausschuss für die Vorstandswahl 2013 ruft alle Mitglieder der Deutschen Gesellschaft für Kartographie e.V. auf, sich durch Brief- oder Präsenzwahl an der Wahl eines neuen Vorstandes der Gesellschaft zu beteiligen. Von den Mitgliedern der DGfK sind der Präsident, zwei Vizepräsidenten (national und international), der Schriftführer und der Schatzmeister zu wählen (§ 10 Abs. 3 Satzung der DGfK). Die Unterlagen für die Briefwahl können die Mitglieder ab einem noch festzulegenden Zeitpunkt von der Website der DGfK (www.dgfk. net) herunterladen oder sie über ihre Sektionen anfordern. Mitglieder der DGfK, die keiner Sektion angehören, können ihre Briefwahlunterlagen zudem schriftlich beim Vorsitzenden des Wahlausschusses, Dipl.-Ing. Fritz Meier, Technische Universität München, Lehrstuhl für Kartographie, D-80290 München anfordern.

Gleichzeitig mit diesem Wahlaufruf bitte ich um Kandidatenvorschläge, die direkt an mich oder an den betreffenden Sektionsleiter gesandt werden sollen.

Der letzte Absendetermin für die Wahlbriefe ist Mittwoch, der 21. August 2013. Die Präsenzwahl findet im Rahmen des 61. Deutschen Kartographentages 2013 in Dresden statt. Das Wahlbüro ist am 28. August 2013 in der Zeit zwischen $11.00 \mathrm{Uhr}$ und 14.00 Uhr geöffnet. Die Bekanntgabe der Wahlergebnisse erfolgt im Rahmen der Mitgliederversammlung, die um 14.00 Uhr beginnt.

Fritz Meier, München

Vorsitzender des Wahlausschusses

DGfK-Exkursion

in den Mittleren Osten

\section{Ankündigung}

Nach erfolgreicher Exkursion der DGfK-Sektion Halle-Leipzig im Februar 2013 nach Marokko, mit solch interessante Stationen wie Casablanca, Tanger, Fes, Quarzazate, aber auch verschiedenen Besonderheiten im Atlas und der Sahara, plant unser langjährig erfahrener Exkursionsleiter Prof. Max Linke für März oder April 2014 unsere nächste kartographische Fachexkursion mit dem Ziel Iran. Die Route wird über 16 Tage von Teheran zunächst an den Kaspisee führen, danach über Täbris an den Urmiasee, von dort weiter in das östliche Shatt el-Arab Delta, weiter über Busher-Shiraz-Yasd-Isfahan-Ghom zurück nach Teheran. In Teheran steht das National Cartographic Centre (NCC) als das führende und zentrale Institut für die iranische Landesaufnahme auf dem Programm. 